\title{
The Onset and Trend of Anisotropy in Greater Ughelli of Niger Delta
}

\author{
${ }^{1}$ Emudianughe.E.J, ${ }^{2}$ Ebeniro.J.O, ${ }^{3}$ Ehirim. C. N., \\ ${ }^{1}$ Federal University of Petroleum Resources, Effurun, Nigeria \\ ${ }^{2,3}$ University of Port Harcourt, Port Harcourt, Nigeria. emusjul@yahoo.co.uk
}

\begin{abstract}
A rock material is anisotropic if it's measurable physical properties such as velocity exhibit different values when measured in different directions. The safest assumption is that the subsurface is anisotropic. If anisotropy is ignored when imaging the subsurface, it could have adverse effects. These adverse effects include sub-optimal time-to-depth conversion, well-to-seismic mismatch, as well as a poorly focused seismic image. This paper presents the result of a seismic anisotropy study carried out on pre-stack depth migrated gathers in Greater Ughelli of the Niger Delta which is generally characterized by repeated sequence of finely layered sand/shale intercalations. These sand/shale sequences are generally bound to be transversely anisotropic. The signature of anisotropy on the depth migrated gathers is the so-called "hockey sticks". The first hockey sticks on pre-stack depth migrated gathers were observed and used for the mapping. The onset of anisotropy was correlated across an entire field (Assa North) in Greater Ughelli depobelt using seismic depth structure maps and fault polygons. The onset of anisotropy ranges from 1400ms - 1600ms. Anisotropy trend in study area is shallow in the NE, of average depth at the middle of the field and very deep SW.
\end{abstract}

Keywords: Anisotropy, Depobelt, Hockey sticks, Sand/Shale Sequence, Sub-optimal

\section{Introduction}

The term anisotropy as applied to seismic implies changes in sound speed as a function of propagation direction. For example, if energy propagates vertically, it moves with a different speed than if it propagates horizontally at the same point in a medium. In general, for layered media, such as interbedded sands and shales, or media that have plate-like mineral grains, sound waves propagate more slowly perpendicular to the layers (or grains) than they do parallel to the layers (or grains). Seismic anisotropy not considered during long offset seismic processing results in poor focused images and laterally mis-positioning of seismic events.

Although the mathematical development of anisotropy has experienced tremendous progress, still the assumption of isotropy is prevalent. Interpretation and processing of these data requires an understanding of the possible anisotropy in the subsurface.

Directional dependence of wave speeds in a medium indicates large-scale order in a material such as layering, alignment of minerals, ordered structures and flow. The two major factors which cause anisotropy are lattice-preferred orientation (which includes the orientation of crystal lattices and intrinsic property of the minerals) and shape-preferred orientation (which include extrinsic property such as structural property, stack of rock layers with different characteristics (Fig 1). Aligned cracks and structural boundaries are the basis of seismic anisotropy.

Several parameters have been estimated and even derived to help correct for seismic anisotropy (Thomsen 1986). Eta $(\eta)$ is one of the parameters used when calculating or correcting for anisotropy. It is the anisotropic combination of $\varepsilon$ and $\delta$, it determines the magnitude of the deviation from hyperbolic moveout (Fig. 2 ) and it is responsible for hockey-sticks displayed on seismic gathers observed at large offset (Fig.3). Eta ( $\eta$ ) can be estimated from surface seismic if one is able to pick the high-order RMO. Another parameter is the delta $(\delta)$, it is the difference between seismic velocity $(\mathrm{Vnmo})$ and true vertical velocity $\left(\mathrm{V}_{0}\right)$. An example of the effect of anisotropy is the lateral shift of reflection point with offset. Figure 2 shows the deflection of the reflection from near offset $\left(15^{0}\right)$ to far offset $\left(45^{\circ}\right)$. During imaging, if anisotropy is not corrected for, targets will definitely be mis-positioned (Vestrum Rob 2002).

Long-offsets reflection curvature becomes non-hyperbolic because of vertical changes in velocity and anisotropy. For this reason non-hyperbolic normal-moveout analysis has to be used. Today we know that the earth is mostly anisotropic. When we estimate velocities in the isotropic case, we are trading offset information for what we think is vertical velocity. 


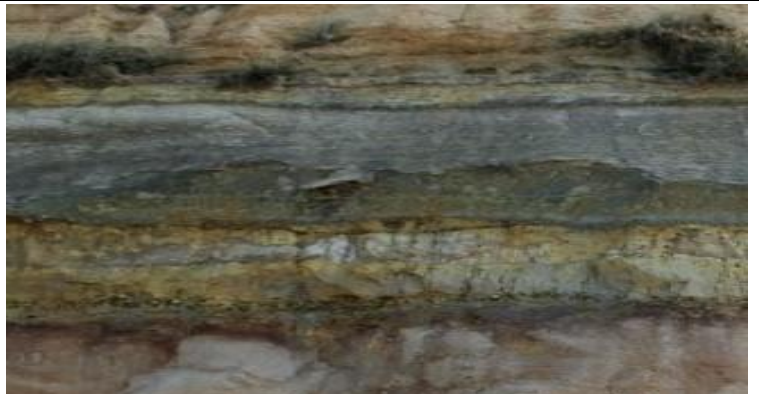

Fig 1: Stack of rock layers with different characteristics geology.um.maine.edu

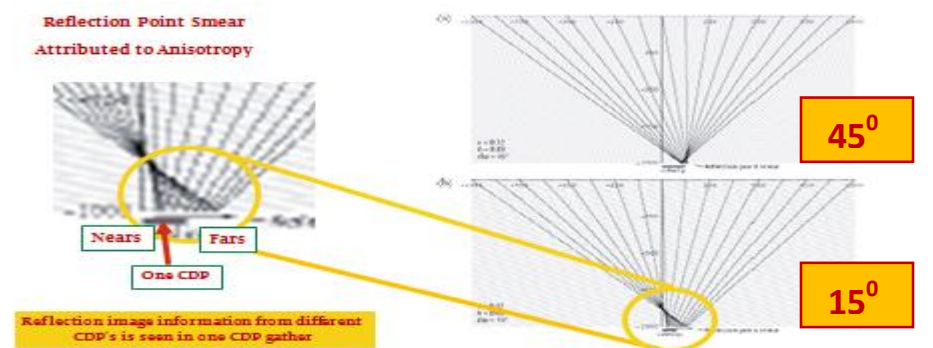

Fig. 2: Lateral shift of reflection point with offset for horizontal reflector beneath anisotropy strata dipping at (a) $45^{0}$ and (b) $15^{\circ}$. (Vestrum Rob, 2002)

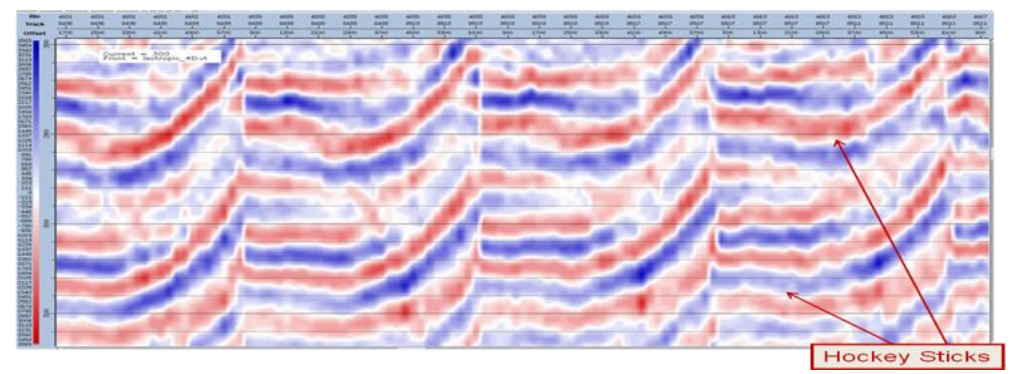

Fig 3: Examples of typical hockey sticks on seismic gathers

Hockey sticks are the signature of anisotropy in the seismic gathers, so they were observed manually and over eleven thousand picks were generated for the purpose of this paper. This paper aims at observing the onset and trend of anisotropy across the depobelt to enable seismic processors get the actual manually mapped onset of anisotropy. This will help in correcting for anisotropy without using computer generated average of the onset of anisotropy. A methodology will be generated for the manual mapping of the onset of anisotropy. This methodology can then be applied in any other field. To the best of my knowledge, manual mapping and the methodology for this mapping has not been done for this field. This enables us to see the trend of anisotropy in this depobelt.

\subsection{Geology of Niger Delta}

The Niger Delta is situated in the Gulf of Guinea and extends throughout the Niger Delta Province as defined by Klett et. al., (1997). From the Eocene to the present, the delta has prograded south-westward, forming depobelts that represent the most active portion of the delta at each stage of its development (Doust and Omatsola, 1990). These depobelts form one of the largest regressive deltas in the world with an area of some $300,000 \mathrm{~km}^{2}$ (Kulke, 1995), a sediment volume of 500,000 km (Hospers, 1965), and a sediment thickness of over $10 \mathrm{~km}$ in the basin depocenter (Kaplan et al, 1994).

The Niger Delta Province contains only one identified petroleum system (Kulke, 1995; Ekweozor and Daukoru, 1994). This system is referred to here as the Tertiary Niger Delta Petroleum System. The maximum extent of the petroleum system coincides with the boundaries of the province. The minimum extent of the system is defined by the real extent of fields and contains known resources (cumulative production plus proved reserves) of 34.5 billion barrels of oil (BBO) and 93.8 trillion cubic feet of gas (TCFG) (14.9 billion barrels of oil equivalent, BBOE) (Petroconsultants, 1996a). Fig 4 shows the location map, this field is located in Greater Ughelli depobelt. 


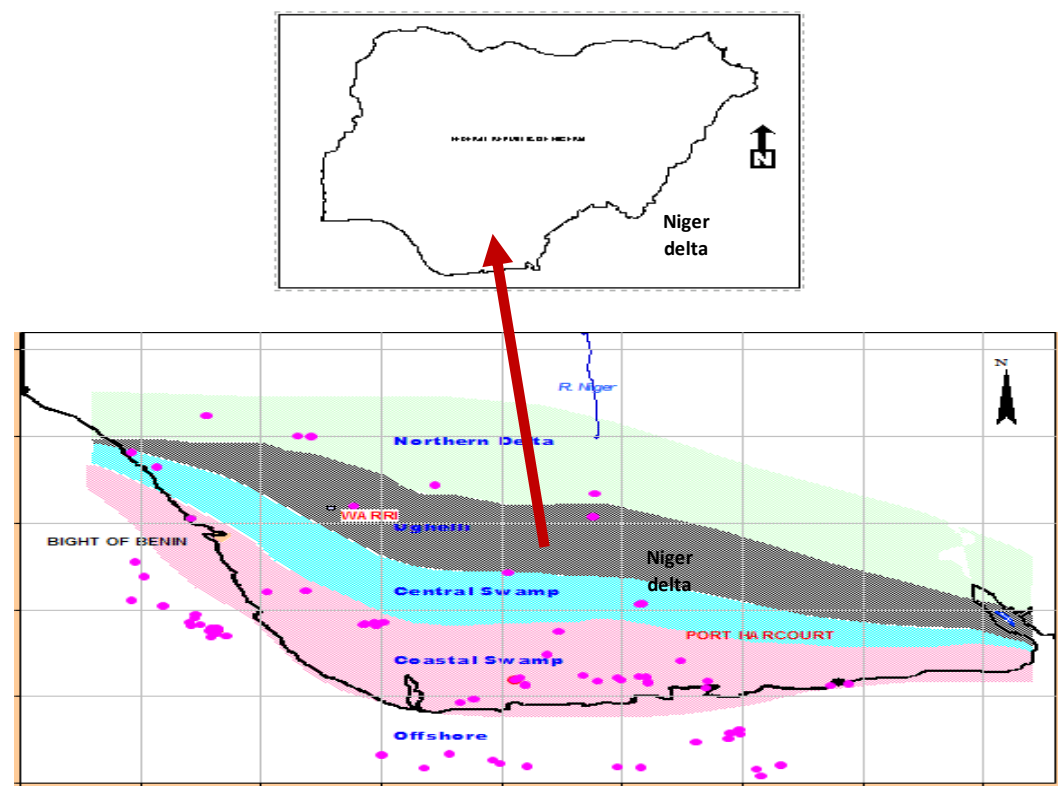

Fig 4: Location map

\section{Methodology}

\subsection{Picking of onset of Anisotropy on depth migrated gathers}

After the pre-processing and NMO corrections, the hockey sticks were observed across an entire field. The first hockey sticks on each track and bin in the seismic gathers were picked (observed) at the interval of $100 \mathrm{~m}$ by $100 \mathrm{~m}$ i.e. every four bins (crossline) and track (inline). In doing this the seismic stack and map view is open for a guide of location and events. This was done for the full volume of Assa North. Over eleven thousand picks were observed. These picks were used to create an Excel spreadsheet. The picks were plotted (Nplot) (Fig 5a.) and imported as event into map view. Since every track and bin were not picked, there was need to smoothened the event for better imaging (Fig 5b). After the smoothening, a map was generated. This is the onset of anisotropy map and was done in both time and depth. This event shows the trend of the onset of anisotropy. From the generated picks; the onset of anisotropy at particular bin (crossline) or track (inline) of choice can be extracted. The work flow used for achieving this onset of anisotropy map can be repeated for any field, and will generate the onset of anisotropy map in that field and equally see the trend of anisotropy in that field.

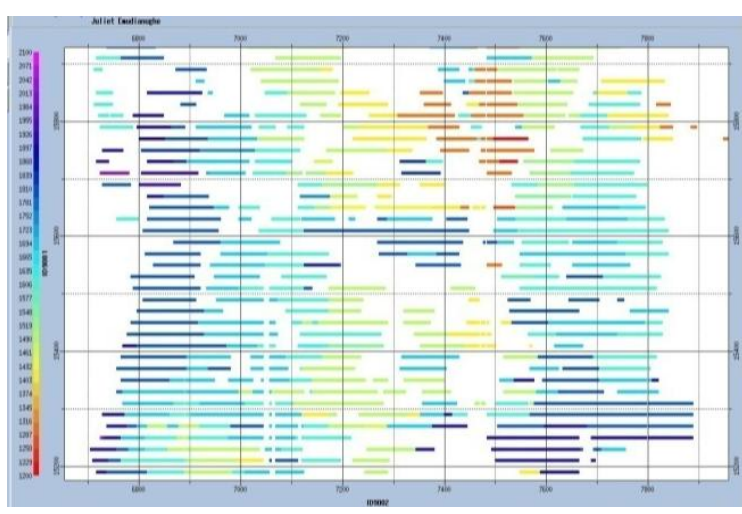

Figure 5a: Generated picks plotted

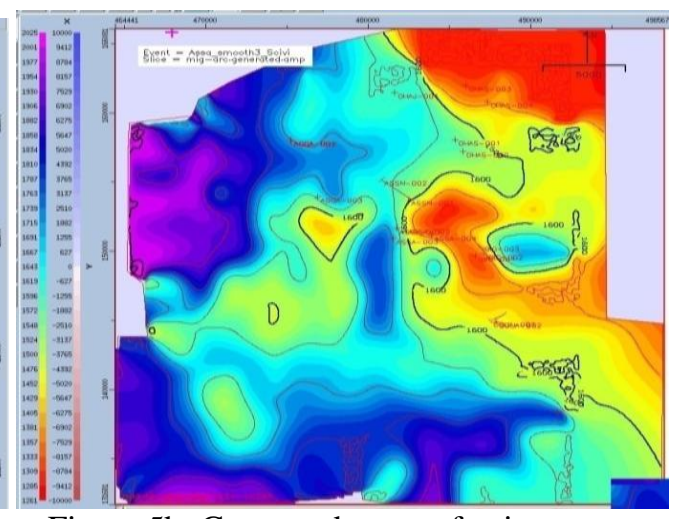

Figure 5b: Generated onset of anisotropy map

\subsection{Trend of Anisotropy in Greater Ughelli}

A trend was clearly seen on the anisotropy generated map. A traverse was taken through the map (fig $5 \mathrm{~b}$ ) to see the trend of the hockey sticks as shown in Figure 6a, 6b and 6c. The onset of anisotropy occurs between $1400 \mathrm{~ms}$ and $1600 \mathrm{~ms}$. The trend of the onset of anisotropy in this depobelt shows that onset is shallow in the North East (NE) and goes deeper in the South West (SW). 


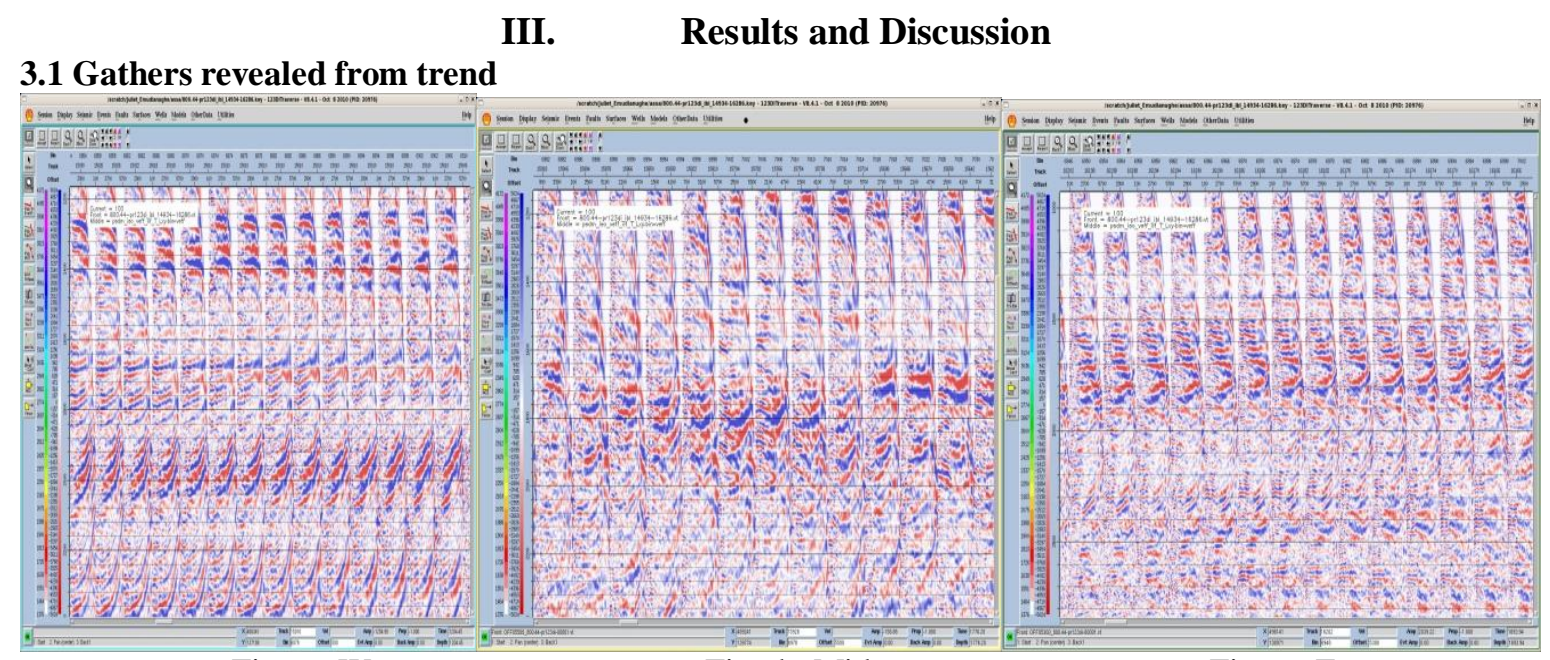

Fig 6a: West

Fig 6b: Mid

Fig 6c: East

Figure $5 \mathrm{~b}$ shows the trend of the onset of anisotropy. A traverse was taken through the anisotropy onset map (Fig $5 \mathrm{~b})$, the traverse revealed a trend, figure $6 \mathrm{a}, 6 \mathrm{~b} \& 6 \mathrm{c}$. On the west of the map the trend shows hockey sticks which are very large (Fig 6a). On the middle of the field the trend shows hockey sticks which are of medium size (Fig $6 \mathrm{~b}$ ). On the East of the map the trend shows hockey sticks which are very small (Fig 6c). This trend reveals that the onset of anisotropy is shallow in the North-East and goes deeper in the South-West.

\subsection{Depth Consideration of the onset of anisotropy}

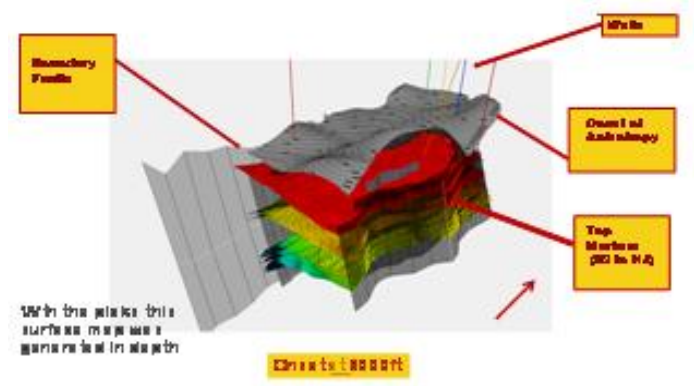

fig 7: Three Dimensional depth surface

This surface helps to understand where the onset occurs amongst other events in the subsurface. It is seen also that there is a dip in the surface (the surface in ash colour in Fig 7) due to the effect of large faults. It is observed also that all the previously interpreted event such as top markers E2 to $\mathrm{H} 4$ which are below the 3D anisotropy depth surface, might likely be under the effect of anisotropy and needs to be corrected for. The onset of anisotropy map was converted to 3D depth surface, so as to have a 3D view of the onset of anisotropy. The arrow in the figure shows direction of north.

\subsection{Assa North Anisotropic Onset Correlation}

The direction of wells (Fig 8a) in order of correlation (Fig 8b)

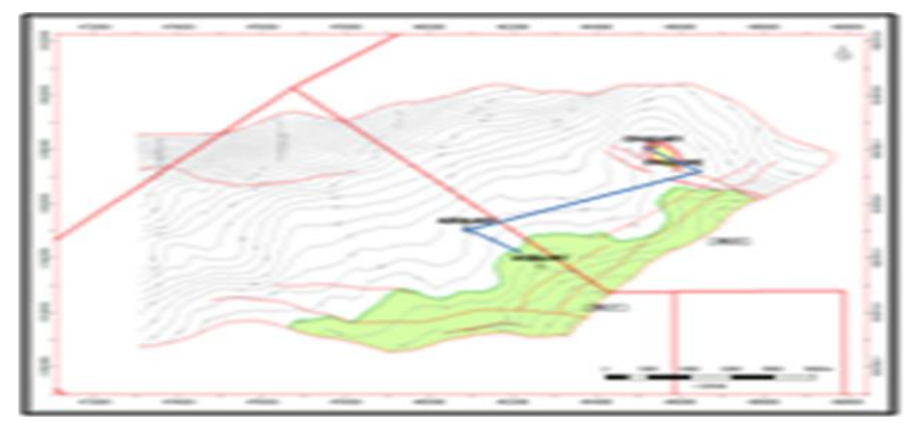

Fig 8a: Direction of correlation well. 


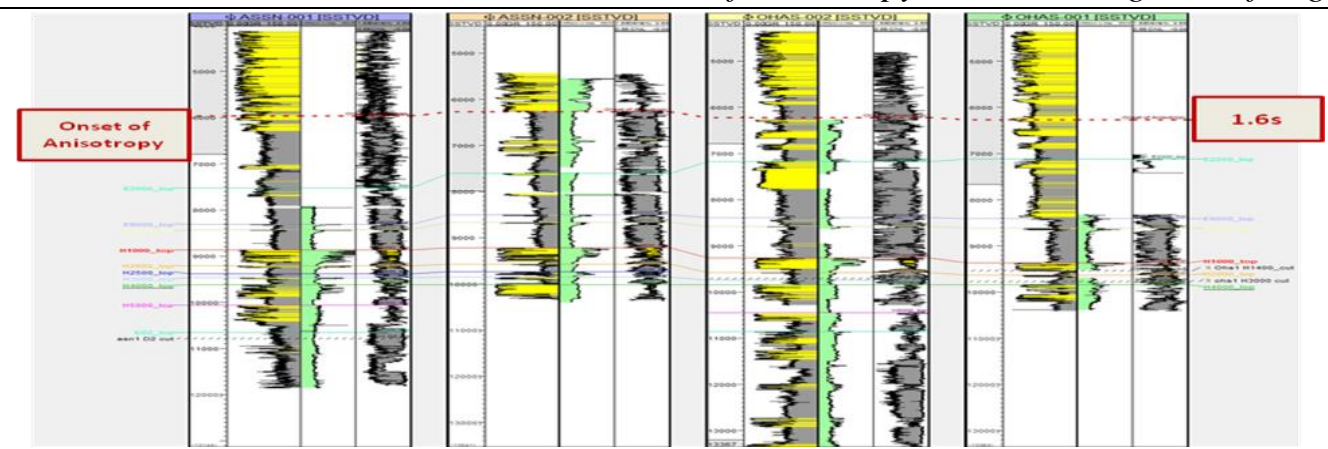

Fig 8b: Well correlation of Assa North 001, 002 and Ohas 001 and 002

Correlating our well $\operatorname{logs}$ to our seismic data, we can obtain a detailed vertical and lateral description of the subsurface. Assa North is a large field with so many wells; Assa North 001, 002, 003004 and Ohaji 001,002 003 and 004. Four wells do not have the complete data for modeling; therefore our model was on four wells. Assa North (ASSN) 001, 002 and OHAS 001 and 002. A correlation was done using gamma ray in this field. The onset of anisotropy generated was then loaded on the well log correlation (Gamma Ray Log) to confirm the picks on the seismic. The anisotropy onset was approximately at same time observed on the seismic session which was at $1600 \mathrm{~ms}(1.6 \mathrm{~s})$. One interesting thing observed was that the onset is at the boundary of sand / shale intercalations. It was neither in the pure shale nor pure sand.

\subsection{Well and Seismic Data Calibration}

A synthetic model was generated (Fig 4.7). Well and seismic data calibration was done, using the synthetic model and the seismic gathers (Fig 3.6). This is to further buttress the accuracy of the picks. The synthetic model and the seismic gathers calibrated, (Fig 4.8a to 4.8c shows that the anisotropy onset occurs at same time.

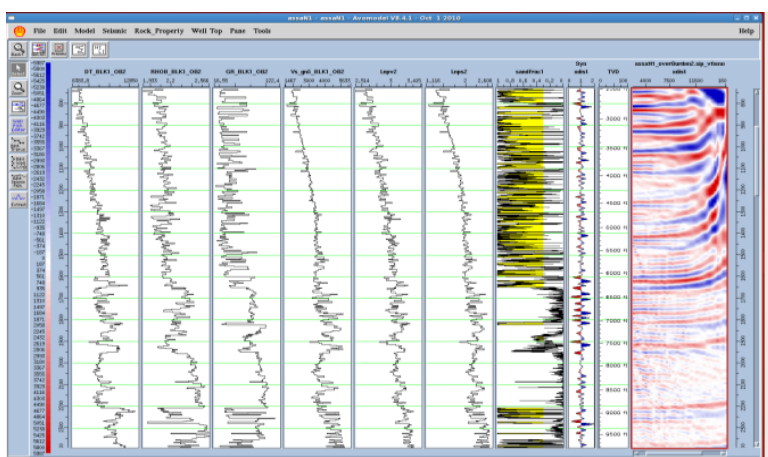

Fig 9a: Well synthetic generated

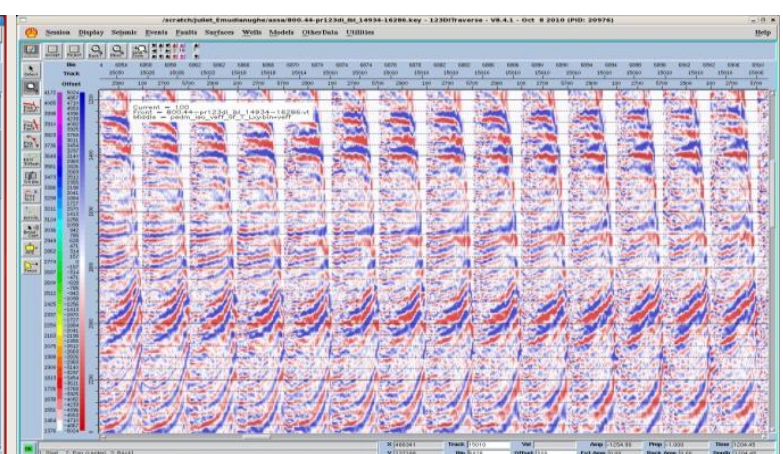

Fig 9b: Seismic gathers

Figure $9 \mathrm{a}$ and $9 \mathrm{~b}$ were displayed side by side on the workstation to see the calibration properly. Due to the effect of smoothened and faults; the trend of the onset of anisotropy is not smooth.

\section{Conclusion}

This paper has been able to manually map the onset of anisotropy which will help seismic processors to better correct for anisotropy. It enables one to extract an onset of a particular inline or crossline of choice instead of taking a computer average. The onset of anisotropy in this depobelts is at $1400 \mathrm{~ms}$ to $1600 \mathrm{~ms}$. This is in an older lithology; Oligocene, thick shale Uvgerinellla (UV 31.3Ma). The onset falls within the zone Paleo 560 in some part of the field. There are limitations in observing picks because it is time consuming (picking over 11000 picks). Secondly picks disappear in area of faults.

Seismic and well synthetic both calibrate to confirm the hockey sticks picked. The anisotropy onset is an NE, SW trend; shallow in NE and deep SW. Generated anisotropy onset surface shows a dip due to effects of major faults. The onset of anisotropy was applied to well log correlation panel, and the onset occurs at the boundaries of sand/shale intercalations. This methodology can be to any field during processing to help in correcting for the effect of anisotropy. 


\section{Acknowledgment}

I appreciate Shell Petroleum Development Company, Nigeria for providing their office premises and workstations for this research. Secondly, Federal University of Petroleum Resources Effurun for their financial support and the leave granted for the purpose of this research.

\section{Reference}

[1] Doust, H., and Omatsola, E., 1990, Niger Delta, in, Edwards, J. D., and Santogrossi, P.A., eds., Divergent/passive Margin Basins, AAPG Memoir 48: Tulsa, American Association of Petroleum Geologists, p. 239-248.

[2] Ekweozor, C. M., and Daukoru, E.M, 1994, Northern delta depobelt portion of the Akata-Agbada petroleum system, Niger Delta, Nigeria, in, Magoon, L.B., and Dow, W.G., eds., The Petroleum System--From Source to Trap, AAPG Memoir 60: Tulsa, American Association of Petroleum Geologists, p. 599-614.

[3] Hospers, J., 1965, Gravity field and structure of the Niger Delta, Nigeria, West Africa: Geological Society of American Bulletin, v. 76, p. 407-422.

[4] Kaplan, A., Lusser, C.U., Norton, I.O., 1994, Tectonic map of the world, panel 10: Tulsa, American Association of Petroleum Geologists, scale 1:10,000,000.

[5] Klett, T.R., Ahlbrandt, T.S., Schmoker, J.W., and Dolton, J.L., 1997, Ranking of the world's oil and gas provinces by known petroleum volumes: U.S. Geological Survey Open-file Report-97-463, CD-ROM.

[6] Kulke, H., 1995, Regional Petroleum Geology of the World. Part II: Africa, America, Australia and Antarctica: Berlin, Gebruder Borntraeger, p. 143-172.

[7] Petroconsultants, 1996a, Petroleum exploration and production database: Houston, Texas, Petroconsultants, Inc., [database available from Petroconsultants, Inc., Houston, TX 77274-0619].

[8] Thomsen 1986, Weak elastic anisotropy, Geophysics. Vol.51. No. Io (October 1986); P. 1954-1966,

[9] Vestrum Rob, 2002, 2D and 3D Anisotropic Depth Migration Case Histories, SEG, Salt Lake City,Utah 2002-1256 October 6 - 11 www.http://geology.um.maine.edu 